\title{
Suppression of the TGF- $\beta /$ Smad signaling pathway and inhibition of hepatic stellate cell proliferation play a role in the hepatoprotective effects of curcumin against alcohol-induced hepatic fibrosis
}

\author{
NANZHENG CHEN ${ }^{1}$, QIANQIAN GENG $^{2}$, JIANBAO ZHENG $^{1}$, SAI HE $^{1}$, XIONGWEI HUO $^{1}$ and XUEJUN SUN ${ }^{1}$ \\ Departments of ${ }^{1}$ General Surgery and ${ }^{2}$ Nuclear Medicine, The First Affiliated Hospital of \\ Medical College, Xi'an Jiaotong University, Xi'an, Shaanxi 710061, P.R. China
}

Received March 31, 2014; Accepted July 22, 2014

DOI: $10.3892 /$ ijmm.2014.1867

\begin{abstract}
The hepatoprotective effects of curcumin against alcohol-induced hepatic fibrosis have rarely been discussed and its mechanisms of action in alcohol-induced liver disease remain unknown. In this study, serum alkaline phosphatase (ALP), aspartate aminotransferase (AST) and alanine aminotransferase (ALT) levels were measured to assess hepatic function; histopathological and immunohistochemical observations were used to evaluate pathological and specific molecular changes in liver tissue and flow cytometry was used to detect the apoptosis in cultured hepatic stellate cells (HSCs), the major fibrogenic cells in the liver; PCR and western blot analysis were employed to evaluate the changes in the expression of molecules and signaling pathways. We demonstrate that curcumin alleviates alcoholinduced hepatic fibrosis by affecting the HSCs. We found that the administration of curcumin inhibited alcohol-induced HSC proliferation and even induced HSC apoptosis by stimulating endoplasmic reticulum (ER) stress. We also found that by suppressing the transforming growth factor- $\beta$ (TGF- $\beta$ )/Smad signaling pathway, the administration of curcumin impaired the production of extracellular matrix proteins in alcohol-stimulated HSCs. These results indicate that curcumin exerts its hepatoprotective effects against alcohol-induced hepatic fibrosis by inhibiting the proliferation and inducing the apoptosis of HSCs by stimulating ER stress and deactivating HSCs by suppressing the TGF- $\beta /$ Smad signaling pathway.
\end{abstract}

\section{Introduction}

Various diseases, including hereditary hepatic metabolic disorders, hepatic viral infection, autoimmune disorder, as well as

Correspondence to: Dr Xuejun Sun, Department of General Surgery, The First Affiliated Hospital of Medical College, Xi'an Jiaotong University, 277 West Yanta Road, Xi'an, Shaanxi 710061, P.R. China

E-mail: sxjsky@aliyun.com

Key words: curcumin, alcohol-induced hepatic fibrosis, hepatic stellate cells chemical agents, such as alcohol can lead to hepatic injury. As a result of alcohol addiction, long-term alcohol consumption becomes one of the leading causes of chronic hepatic disease (1). It has been reported that the risk of developing hepatitis and hepatic fibrosis increases significantly if an individual ingests alcohol everyday (60-80 $\mathrm{g}$ for males and $20 \mathrm{~g}$ for females) in over an extented period of time (2). As the prequel to cirrhosis, hepatic fibrosis is characterized by excessive extracellular matrix (ECM) generation, which impairs normal hepatic function (3). There are positive correlations between hepatic fibrosis and hepatic cirrhosis/hepatic cellular carcinoma (HCC), which are the leading causes of mortality in patients with liver diseases (4).

In response to hepatic damage, hepatic stellate cells (HSCs), which take charge of hepatic ECM secretion and deposition, are often activated (5). The activation of HSCs is characterized by the transformation of HSCs from a quiescent to a proliferative and profibrogenic state (6). It is believed that activated HSCs are the main source of collagen and tissue inhibitors of metalloproteinase (TIMPs) during hepatic fibrosis (7). Although multiple factors may participate in HSC activation, including growth factors, inflammatory factors and free radicals, evidence strongly suggests an association with the transforming growth factor- $\beta$ (TGF- $\beta$ ) signaling pathway (8). The TGF- $\beta / \mathrm{Smad}$ signaling pathway is activated by TGF- $\beta$ binding to its receptors located on the cell membrane. The downstream proteins, namely Smad2 and Smad3, are activated by phosphorylation (9), which further promotes the transcription of genes conducting ECM protein synthesis (10). Additionally, in this progress, another Smad protein, Smad7, acts as the inhibitor of the TGF- $\beta$ /Smad signaling pathway through its ubiquitinproteasome degradation activity (11).

Since ancient times, herbal medicine has been used in China and India. Some effective ingredients extracted from herbs, such as baicalein, emodin, ginsenoside and curcumin have become hot research topics due to their efficacy against diseases, such as inflammatory diseases, cancer, viral infections, ischemia and chronic hepatic disease (12-15). Curcumin, also known as 1,7-bis(4-hydroxy-3-methoxyphenyl)-1,6-heptadiene-3,5dione, is an important phytochemical extracted from a herbal plant known as turmeric (Curcuma longa Linn.). Curcumin 
possesses a variety of biological properties, including antioxidant, anti-carcinogenic and anti-inflammatory properties (16). The hepatoprotective effects of curcumin against hepatic fibrosis have previously been demonstrated (17). Furthermore, it has been demonstrated that the mechanisms of action of curcumin in inhibiting hepatic fibrosis correlate with the inhibition of the TGF- $\beta /$ Smad signaling pathway (18). However, to the best of our knowledge, the majority of studies on the anti-fibrotic effects of curcumin have been based on carbon tetrachloride-induced hepatic fibrosis, which does not mimic the toxicity of alcohol specifically. Moreover, there are limited studies available that have focused on the effects of curcumin on HSCs incubated in alcoholic conditions; these cells may be one of the critical mechanisms through which curcumin exerts its effects against hepatic fibrosis.

In the present study, we assessed and investigated the capacity and possible mechanisms of action of curcumin in alleviating alcohol-induced hepatic fibrosis in rats. Additionally, an in vitro investigation was also conducted to evaluate the effects of curcumin on the alcohol-induced activation of rat HSCs. Curcumin may be considered as a possible therapeutic target in alcohol-induced hepatic fibrosis.

\section{Materials and methods}

Animal grouping and treatment. Thirty male Sprague-Dawley (SD) rats (6 weeks old; mean body weight, 205 $\pm 25 \mathrm{~g}$ ) purchased from the Animal Experimental Center of Xi'an Jiaotong University, Xi'an, China were used in this study. The animals were raised in separate polypropylene cages for 1 week prior to treatment under controlled conditions (artificial 12/12 h-cycle; mean temperature of $22 \pm 1^{\circ} \mathrm{C}$; humidity at $65 \pm 4 \%$ ). The animal experimental procedures were carried out strictly in accordance with the Guide for the Care and Use of Laboratory Animals of the National Institutes of Health $(\mathrm{NIH})$ and approved by the Medical Animal Research Ethics Committee of Xi'an Jiaotong University. The rats were divided into 3 groups evenly and randomly, including a control group $(\mathrm{Ctrl}, \mathrm{n}=10)$, alcohol group (Alc, $n=10$ ) and alcohol + curcumin group (Alc + Cur, $n=10)$. Hepatic fibrosis was induced by the oral administration of alcohol as described in a previous study (19). Rats in the Alc group received an oral administration of ethanol solution [ $30 \% \mathrm{v} / \mathrm{v}$, intragastric (i.g.), $10 \mathrm{ml} / \mathrm{kg} / \mathrm{day}$ ] for 4 weeks; rats in the $\mathrm{Alc}+\mathrm{Cur}$ group received an oral administration of ethanol solution (30\% v/v, i.g., $10 \mathrm{ml} / \mathrm{kg} /$ day) for 4 weeks and then curcumin solution [suspended in phosphate-buffered saline (PBS), i.g., $200 \mathrm{mg} / \mathrm{kg}, 300 \mathrm{mg} / \mathrm{kg}$ body weight) for 12 weeks (20).

Assessment of hepatic function. Whole blood sample from rats were collected by femoral artery puncture when the rats were sacrificed by an overdose of chloral hydrate by intraperitoneal injection $(10 \% \mathrm{v} / \mathrm{v})$. The samples were delivered to the Clinical Laboratory at the The First Affiliated Hospital of Medical College, Xi'an Jiaotong University. The activities of alkaline phosphatase (ALP), aspartate aminotransferase (AST) and alanine aminotransferase (ALT) in serum were detected using an enzyme coupling rate method.

Histopathological and immunohistochemical evaluation. Immediately after harvesting, hepatic tissue was fixed by neutral buffered formalin (10\% v/v, $\mathrm{pH} 7.4)$, embedded in paraffin and then sectioned at a thickness of $4 \mu \mathrm{m}$. Tissue slices were stained with fibrosis-specific staining, Sirius red staining, in accordance with the protocols described in previous studies $(21,22)$. The prepared sections were also subjected to immunohistochemical staining by incubation with antibodies against $\alpha$-smooth muscle actin ( $\alpha$-SMA) and collagen-I (Coll-I) (both from Abcam, Cambridge, UK). After images were acquired, the quantification of the positively stained areas expressed as optical density (OD) was performed using ImageJ software (version $1.43 \mathrm{~b}$; $\mathrm{NIH}$, Bethesda, MD, USA).

Cell culture and treatments. HSCs were isolated from the livers of the SD rats (body weight, $101 \pm 7 \mathrm{~g}$ ). The isolation procedures were implemented following previously described protocols (23). The purity of the isolated HSCs was evaluated by immunohistochemistry using antibody against desmin (Abcam) and intrinsic vitamin A autofluorescence under a phase-contrast microscope (Model CH20; Olympus, Tokyo, Japan). Cell viability was evaluated by trypan blue exclusion assay. Cell purity and viability exceeded $90 \%$. The cells were maintained in culture flasks (Corning, Inc., Corning, NY, USA) containing minimum essential medium (MEM) supplemented with 5\% fetal calf serum (FCS) (both from Gibco, Carlsbad, CA, USA), $1 \%$ non-essential amino acid (NEA; HyClone, Logan, UT, USA) and penicillin-streptomycin $(100 \mathrm{U} / \mathrm{ml}$; Invitrogen, Carlsbad, CA, USA) in a humidified atmosphere of $5 \% \mathrm{CO}_{2}$ at $37^{\circ} \mathrm{C}$. After being washed with sterilized PBS, the cells were subcultured after trypsinization twice per week. The effects of curcumin on the HSCs were investigated by pre-treatment with curcumin at serial concentrations $(0,10,20,30,40,50$ and $60 \mu \mathrm{mol} / \mathrm{l})$. Equal amounts of cells were assigned to the Ctrl, Alc and Alc + Cur groups. Cells in the Alc + Cur group were treated with ethanol (50 mmol/l; Sigma-Aldrich (St. Louis, MO, USA) for $24 \mathrm{~h}$ and then treated with curcumin (50 mmol/l; Sigma-Aldrich) for a further $24 \mathrm{~h}$; cells in the Alc group were only treated with ethanol for $24 \mathrm{~h}$. The cells were then cultured in a humidified atmosphere of $5 \% \mathrm{CO}_{2}$ at $37^{\circ} \mathrm{C}$ for $24 \mathrm{~h}$.

Cell viability assay. A colorimetric 3-(4,5-dimethylthiazol2-yl)-2,5-diphenyltetrazolium bromide (MTT) assay was used to assess the cell viability in the present study. Briefly, cells from each group planted in a 96-well culture plate (Corning, Inc.) for $24 \mathrm{~h}$ were washed with sterilized PBS and then incubated with MTT (5 mg/ml; Sigma-Aldrich) for $4 \mathrm{~h}$. After being washed with sterilized PBS, the cells were then dissolved in dimethyl sulfoxide (DMSO; Sigma-Aldrich). The absorbance value at $450 \mathrm{~nm}$ was read using a 96-well plate reader (Bio-Rad, Hercules, CA, USA). The ratio of the number of viable cells in the experimental wells to the control wells was used to express the results of cell viability.

Assessment of cell apoptosis. Cell apoptosis was evaluated by flow cytometry. Cells at a density of $5 \times 10^{3}$ cells/well in each group were collected for the analysis of apoptosis by V-FITC and propidium iodide (PI) (both from Santa Cruz Biotechnology, Inc., Santa Cruz, CA, USA) double staining using a flow cytometer (FACSCalibur; BD Biosciences, Franklin Lakes, NJ, USA) in accordance with the protocol described in a previous study (24). 


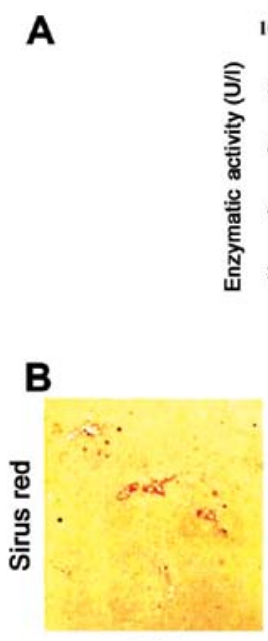

Ctri

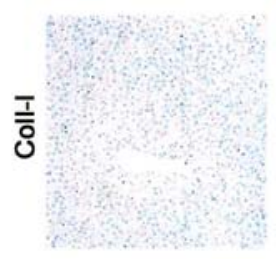

Ctrl

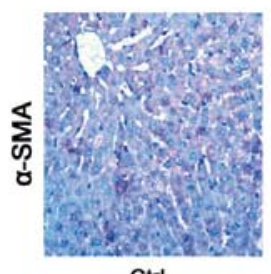

Ctrl
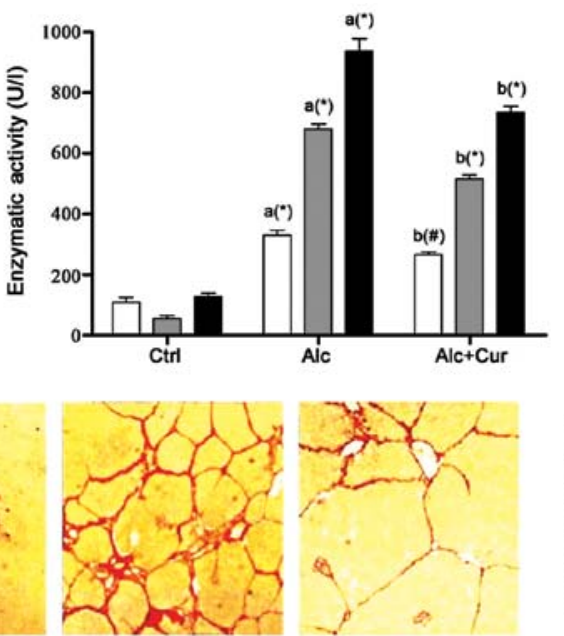

Alc

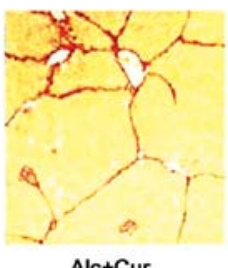

Alc+Cur

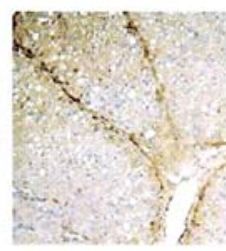

Alc

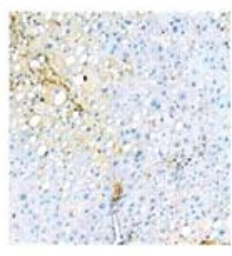

Alc + Cur
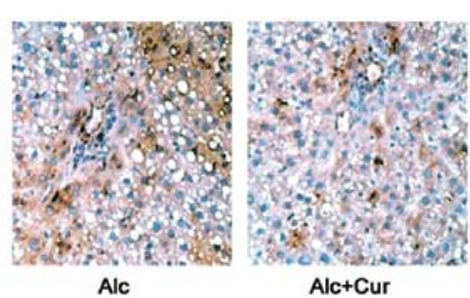
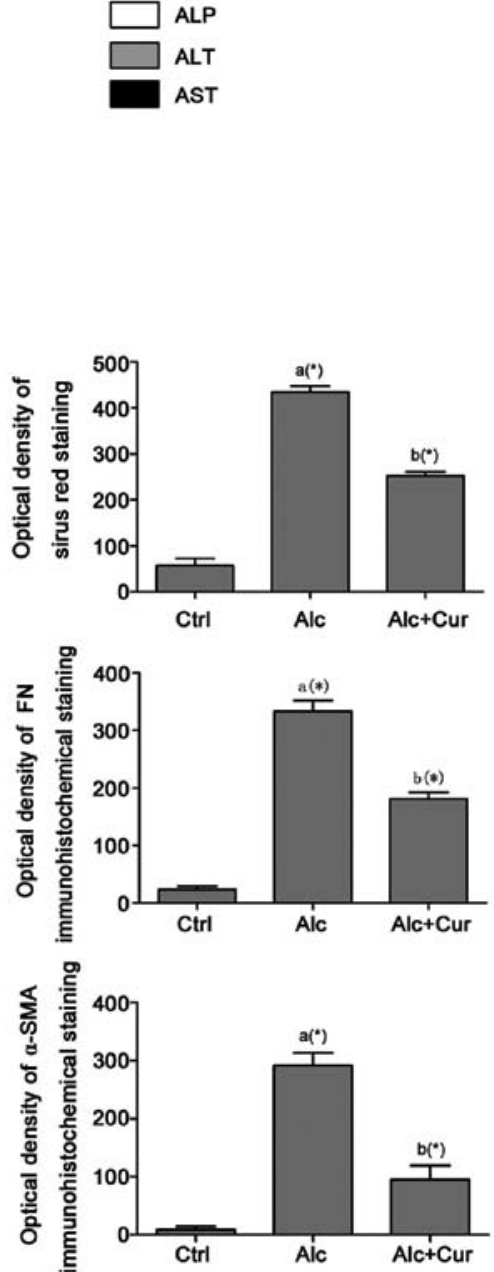

Figure 1. Effects of curcumin on hepatic function and fibrogenesis in rats with alcohol-induced hepatic fibrosis. (A) Ddetected levels of serum alkaline phosphatase (ALP), alanine aminotransferase (ALT) and aspartate aminotransferase (AST) in the rats from the control group (Ctrl), alcohol group (Alc) and alcohol + curcumin group (Alc + Cur). Enzymatic activities of ALP, ALT and AST are presented as columns and as the means \pm standard deviation (SD). (B) Captured images and quantification of Sirius red staining and immunohistochemical staining for collagen-I and $\alpha$-smooth muscle actin ( $\alpha$-SMA) in the sections from the liver tissue in the Ctrl, Alc and Alc + Cur groups. The letter ' $a$ ' indicates significant differences compared to Ctrl; the letter ' $b$ ' indicates significant differences compared to Alc group. ${ }^{*} \mathrm{P}<0.05$ and ${ }^{\#} \mathrm{P}<0.01$.

Real-time reverse transcription-polymerase chain reaction $(P C R)$. From both rat hepatic tissue and cultured HSCs, total RNA was extracted using the RNAfast 200 kit (Fastagen Biotech Co., Ltd., Shanghai, China) then reverse transcription was performed using the PrimeScript RT reagent kit (Takara Bio, Inc., Shiga, Japan). SYBR Premix Ex Taq ${ }^{\mathrm{TM}}$ II (Takara Bio, Inc.) was used to perform real-time PCR and the results were then detected using a PRISM 7500 real-time PCR detection system (Applied Biosystems, Foster City, CA, USA). Oligonucleotide primers for $\alpha$-SMA, collagen-I, TGF- $\beta 1$, fibronectin, Smad7 and glyceraldehyde 3-phosphate dehydrogenase (GAPDH) were synthesized by Takara Bio, Inc. Relative mRNA expression levels were calculated by $\Delta$ cycle threshold $\left(\Delta \mathrm{Ct}=\mathrm{Ct}_{\text {Target }}-\mathrm{Ct}_{\mathrm{GAPDH}}\right)$ and GAPDH was introduced as an internal reference gene.

Western blot analysis. Both the rat hepatic tissue and cultured HSCs were homogenized using lysis buffer $(40 \mathrm{mmol} / 1 \mathrm{Tric}-\mathrm{HCl}$, $150 \mathrm{mmol} / 1 \mathrm{KCl}, 1 \mathrm{mmol} / \mathrm{l}$ EDTA, $100 \mathrm{mmol} / 1 \mathrm{NaVO}_{3}$, $1 \%$ Triton X-100) with PMSF (1 mmol/1; Santa Cruz Biotechnology, Inc.). After concentration detection using the
BCA protein assay kit (Santa Cruz Biotechnology, Inc.), $50 \mu \mathrm{g}$ protein sample were separated by vertical sodium dodecyl sulfate-polyacrylamide gel (10 or 8\%) electrophoresis and then transferred onto PVDF membranes which were subsequently blocked in defatted milk [5\%, dissolved in Tris-buffered saline with Tween-20 (TBST) buffer] to block non-specific binding. Immunoblots were then detected using specific antibodies against collagen-I, fibronectin, $\alpha$-SMA, TGF- $\beta 1$ and proliferating cell nuclear antigen (PCNA) (all purchased from Abcam), Smad3, phospho-Smad3, Smad7 (all from Cell Signaling Technology, Danvers, MA, USA), glucose-regulated protein-78 (GRP-78), CCAAT/enhancer-binding protein homologous protein (CHOP) (both from Abcam) and GAPDH (Santa Cruz Biotechnology, Inc.). After the unbounded antibodies were washed with TBST-Tween-20 (0.02\%), the membranes were incubated with antibody conjugated to HRP (Santa Cruz Biotechnology, Inc.) for $2 \mathrm{~h}$ at $37^{\circ} \mathrm{C}$. Finally, the immunoblots were visualized and determined using ECL western blotting detection reagent (Amersham Pharmacia Biotech, Piscataway, NJ, USA). ImageJ software (version 1.43b; NIH) was then used to determine and quantify the density of the bands. 
A

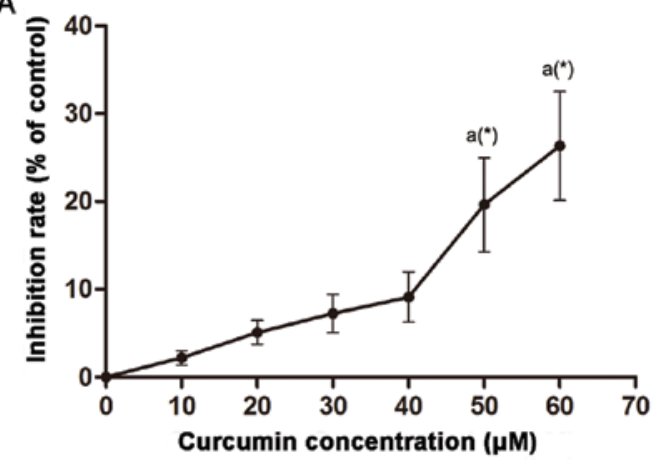

B

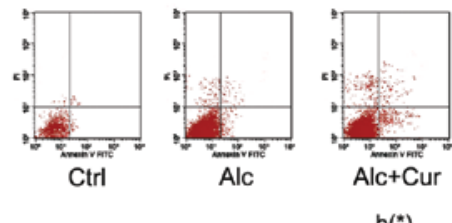

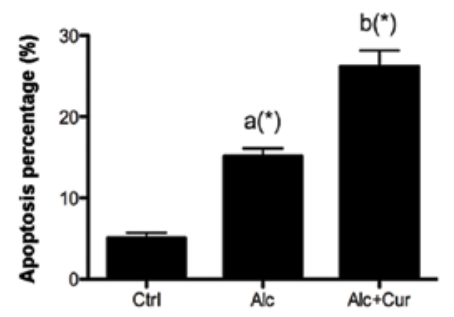

C

D

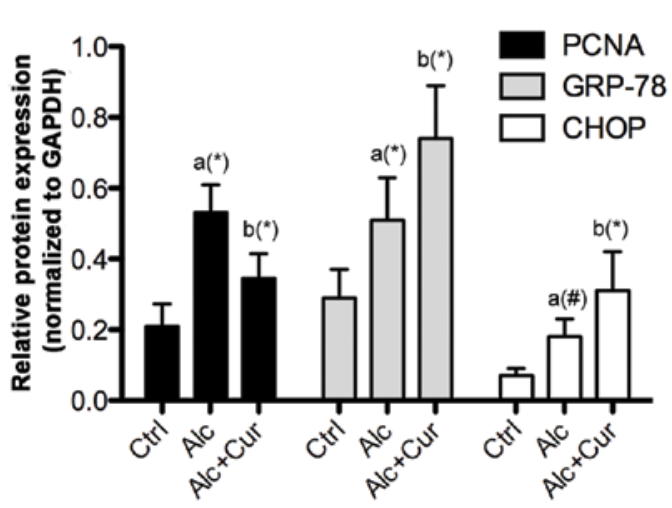

Figure 2. Effects of curcumin on alcohol-stimulated hepatic stellate cell (HSC) proliferation and endoplasmic reticulum (ER) stress. (A) Results of MTT assay. The inhibitory effects of curcumin on HSC proliferation were concentration-dependent. (B) Apoptosis of HSCs in the control group (Ctrl), alcohol group (Alc) and alcohol + curcumin group (Alc + Cur). The apoptosis percentage is presented as columns as the means \pm standard deviation (SD). (C) Immunoblots of proliferating cell nuclear antigen (PCNA), glucose-regulated protein-78 (GRP-78), CCAAT/enhancer-binding protein homologous protein (CHOP) and glyceraldehyde 3-phosphate dehydrogenase (GAPDH) in the HSCs from the Ctrl, Alc and Alc + Cur groups. (D) Relative protein expression levels of PCNA, GRP-78 and CHOP in the HSCs in the Ctrl, Alc and Alc + Cur groups. Bars represent the mean \pm standard deviation (SD). The letter 'a' indicates significant differences compared to $\mathrm{Ctrl}$; the letter ' $\mathrm{b}$ ' indicates significant differences compared to Alc group. ${ }^{*} \mathrm{P}<0.05$ and ${ }^{\#} \mathrm{P}<0.01$.

Statistical analysis. Data collected in this study are expressed as the means \pm standard deviation (SD). All statistic analyses were performed using SPSS software (version 15.0; SPSS Inc., Chicago, IL, USA). Differences in parameters among the groups were analyzed by one-way analysis of variance followed by the Nweman-Keuls post-hoc test. Differences were considered statistically significant with P-values $<0.05$.

\section{Results}

Curcumin alleviates alcohol-induced hepatic functional damage and fibrosis in vivo. The administration of alcohol for 4 weeks significantly impaired hepatic function, which was evidenced by the marked increase in serum ALP, ALT and AST levels in the Alc group compared with the Ctrl group (Fig. 1). By contrast, treatment with curcumin (Alc + Cur group) resulted in a marked decrease in the serum levels of AST and ALT compared with the Alc group. The therapeutic effects of curcumin against hepatic fibrosis were then examined by histopathological and immunohistochemical staining. Sirius red staining was used to stain hepatic collagen fiber. The optical densities of the positively stained areas in Sirius red staining, and collagen-I staining increased significantly in the Alc group compared with the Ctrl group. However, following treatment with curcumin, the densities of the positively stained areas in collagen-I and Sirius red staining decreased significantly in the Alc + Cur group compared with the Alc group. Moreover, stimulation with alcohol induced a significant increase in the density of $\alpha$-SMA-positive staining, which suggests the activation of the HSCs in the Alc group compared with the Ctrl group. The inhibitory effects of curcumin against HSC activation were demonstrated by the marked decrease in the density of $\alpha$-SMA-positive staining in the Alc + Cur group.

Curcumin inhibits HSC activation by suppressing HSC proliferation and inducing apoptosis through the enhancement of endoplasmic reticulum (ER) stress in vitro. The proliferation of the HSCs was promoted by incubation with alcohol as evidenced by the observation of the increased expression of PCNA (Fig. 2). Following pre-treatment with serial concentrations of curcumin, the proliferation of the alcohol-stimulated HSCs was inhibited, as evidenced by the decrease in cell viability and the decreased PCNA expression. The results from MTT cell viability assay indicated that treatment with curcumin at a concentration of $50 \mu \mathrm{M}$ markedly inhibited the proliferation of the alcohol-stimulated HSCs. Furthermore, treatment with curcumin markedly increased the apoptosis of the alcohol-stimulated HSCs through ER stress signaling, which was evidenced by the elevated apoptotic rate detected by flow cytometry and the increased expression of GRP-78 and CHOP. 

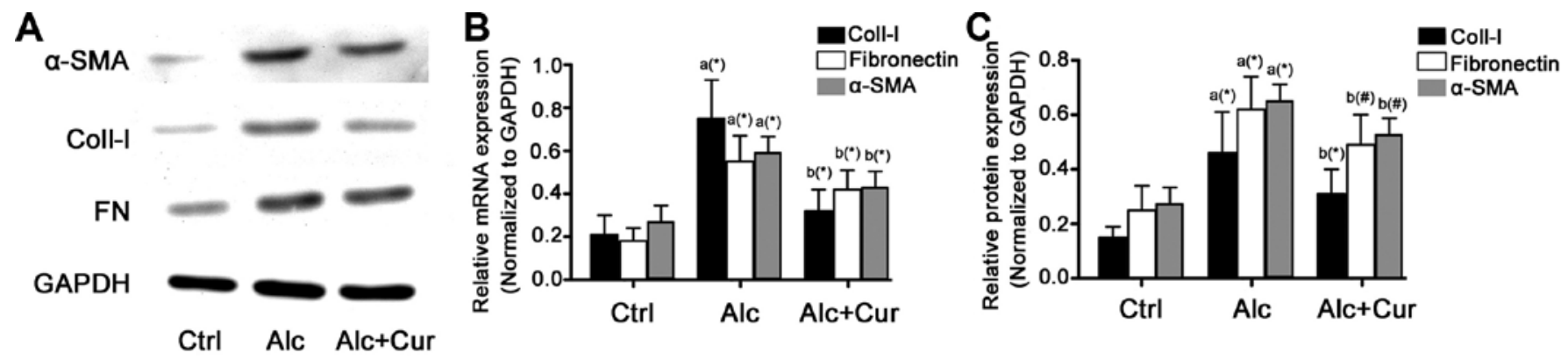

Figure 3. Effects of curcumin on fibrogenesis and activation of alcohol-stimulated hepatic stellate cells (HSCs). (A) Immunoblots of $\alpha$-smooth muscle actin ( $\alpha$-SMA), collagen-I (Coll-I), fibronectin and glyceraldehyde 3-phosphate dehydrogenase (GAPDH) in the HSCs from the control group (Ctrl), alcohol group (Alc) and alcohol + curcumin group (Alc + Cur). (B) Quantification of relative mRNA expression of $\alpha$-SMA, collagen-I and fibronectin in HSCs by polymerase chain reaction (PCR) from the Ctrl, Alc and Alc + Cur groups. (C) Quantification of relative protein expression of $\alpha$-SMA, collagen-I and fibronectin. The letter ' $a$ ' indicates significant differences compared to $\mathrm{Ctrl}$; the letter ' $\mathrm{b}$ ' indicates significant differences compared to Alc group. ${ }^{*} \mathrm{P}<0.05$ and ${ }^{\#} \mathrm{P}<0.01$.
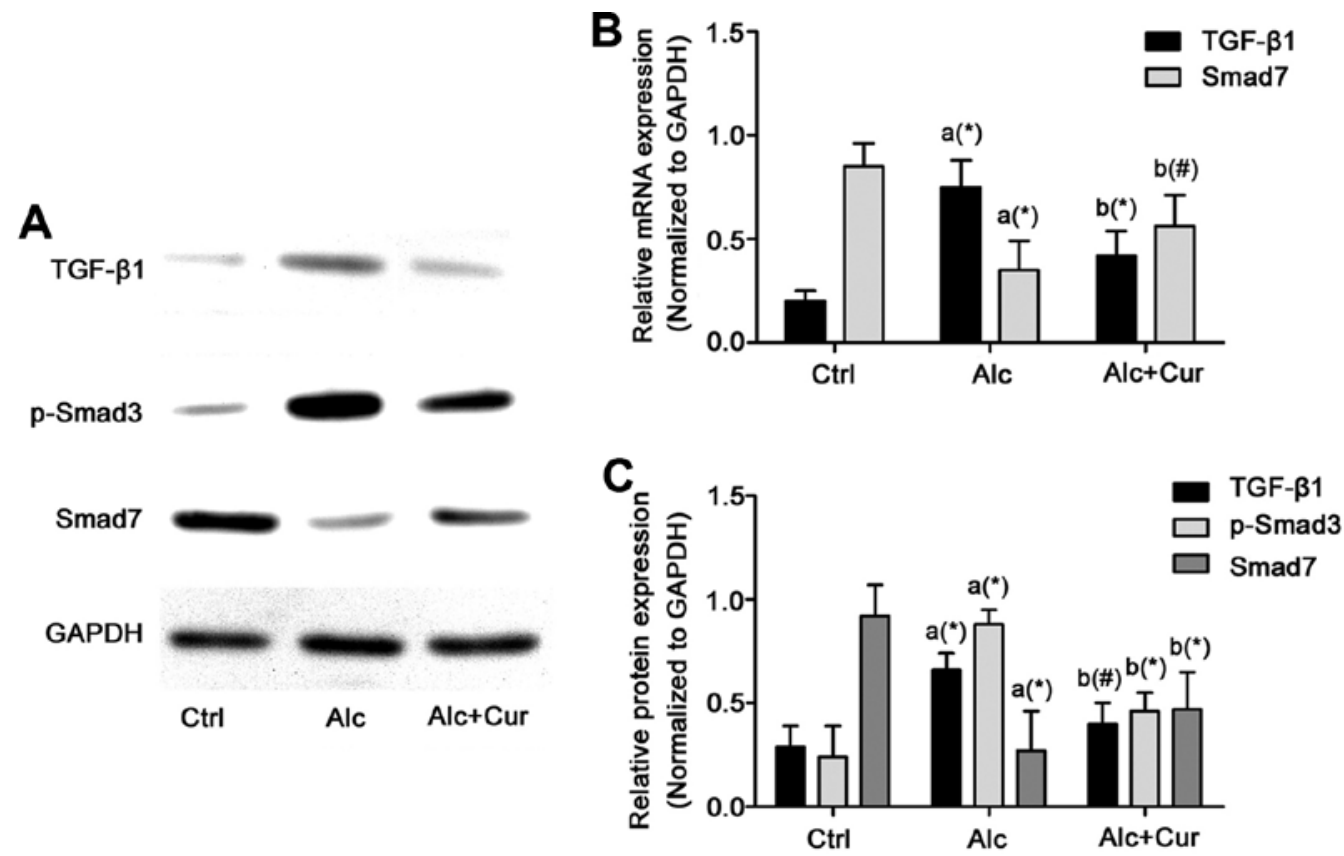

Figure 4. Effects of curcumin on the transforming growth factor- $\beta$ (TGF- $\beta$ )/Smad signaling pathway in alcohol-stimulated hepatic stellate cells (HSCs). (A) Immunoblots of TGF- $\beta 1$, p-Smad3, Smad7 and glyceraldehyde 3-phosphate dehydrogenase (GAPDH) in the HSCs from the control group (Ctrl), alcohol group (Alc) and alcohol + curcumin group (Alc + Cur). Quantification of these immunoblts is shown in (C); columns represent the mean \pm standard deviation (SD). (B) Relative mRNA expression of TGF- $\beta 1$ and Smad7 in HSCs from the Ctrl, Alc and Alc + Ctrl groups by polymerase chain reaction (PCR). The letter ' $a$ ' indicates significant differences compared to Ctrl; the letter ' $\mathrm{b}$ ' indicates significant differences compared to Alc group. "P<0.05 and ${ }^{\#} \mathrm{P}<0.01$.

Curcumin deactivates HSCs and impaires the expression of collagen-I and fibronectin in alcohol-stimulated HSCs in vitro. The expression of $\alpha$-SMA, collagen-I and fibronectin at both the transcriptional and translational levels markedly increased following incubation of the cells with alcohol (Fig. 3). However, following the administration of curcumin, the mRNA and protein expression of $\alpha$-SMA, collagen-I and fibronectin markedly decreased.

Curcumin deactivates the TGF- $\beta$ signaling pathway. The protein expression of TGF- $\beta$ and phosphorylated Smad3 markedly increased, while that of Smad7 markedly decreased following the administration of alcohol in the HSCs (Fig. 4). However, following treatment with cucumin (Alc + Cur group), the TGF- $\beta$ signaling pathway was markedly inhibited, which was reflected by the increased expression of Smad7 and the decreased expression of TGF- $\beta$ and phosphorylated Smad3 compared with the Alc group. Compared with the Alc group, Smad7 expression increased, while that of TGF- $\beta$ and phosphorylated Smad3 markedly decreased in the isolated HSCs treated with curcumin.

\section{Discussion}

As one of the hepatotoxic agents, alcohol abuse results in liver damage, which eventually leads to progressive and chronic hepatic fibrosis, a process through which excessive collagenrich ECM is generated to replace normal hepatic tissue (15). 
Hepatic fibrosis progresses and develops into hepatic cirrhosis, whose association with hepatic failure, hepatocellular carcinoma and portal hypertension has been confirmed (25). The progression of hepatic fibrosis into cirrhosis may be reversible during the early stages (26), thus preventing chronic liver disease.

Curcumin, also referred to as diferuloylmethane, is extracted from the rhizome of Curcuma longa Linn. which as long been used in Chinese and Indian traditional medicine (27). Curcumin has attracted the attention of researchers due to its potential therapeutic effects in the treatment of carcinomas, inflammation, asthma, diabetes and hepatic failure (28). Studies have reported that curcumin attenuates the development of liver fibrosis in various animal models $(29,30)$. In the present study, we demonstrate the hepatoprotective effects of curcumin in a rat model of hepatic fibrosis induced by the oral administration of alcohol. We demonstrate that curcumin alleviates the alcohol-induced loss of hepatic function. Furthermore, our results demonstrated that curcumin reduced collagen generation in hepatic tissue, which was stimulated following the administration of alcohol. These results from our in vivo study suggest and confirm that curcumin mitigates alcohol-induced hepatic fibrosis.

It is believed that HSCs are the major source of the components of the ECM, including collagen, fibronectin and other matrix proteins contributing to the genesis and development of hepatic fibrosis (31). HSCs in a quiescent state are activated by hepatic injury which is induced by a number of agents, including alcohol. In such circumstances, activated HSCs are characterized by proliferation, contractility and fibrogenesis, which are marked by the increased expression of $\alpha$-SMA, according to both in vivo and in vitro studies $(32,33)$. In this study, the observation of the decreased hepatic $\alpha$-SMA expression following treatment with curcumin in rats administered alcohol suggests that the deactivation of HSCs plays an important role in the hepatoprotective effects of curcumin against fibrosis.

Thus, the deactivation and inhibition of HSCs has become a critical and potential therapeutic target in the treatment of hepatic fibrosis. The ER is a cell organelle conducting protein folding and handling calcium homeostasis (34). ER stress occurs in response to harmful stimuli. Prolonged and overwhelming ER stress activates the downstream apoptotic pathway through the activation of pro-apoptotic molecules, such as CHOP (35). Our data revealed that the proliferation of HSCs was stimulated following incubation of the cells with alcohol, as evidenced by the increased expression of PCNA and increased cell viability (MTT assay). We also demonstrated that curcumin induced the apoptosis of the alcohol-stimulated HSCs, which resulted in the inhibition of proliferation. The expression of GRP-78, a molecular marker of ER stress (36), and CHOP increased significantly following treatment with curcumin in the alcoholstimulated HSCs. These results suggest that curcumin inhibits the proliferation and induces the apoptosis of alcohol-stimulated HSCs by activating ER stress.

It is believed that TGF- $\beta$ is among the most potent cytokines that initiate the generation of myofibrobalsts (37). Studies have demonstrated that the activation of the TGF- $\beta /$ Smad signaling pathway is responsible for the progression of hepatic fibrosis following liver injury (38). TGF- $\beta$ stimulates its downstream Smad proteins through tansmembrane receptors, and the transcriptions of several genes that encode ECM components, such as collagen and fibronectin are then activated (39). In this study, an in vitro experiment was implemented to investigate the effects of curcumin on the TGF- $\beta /$ Smad signaling pathway in an animal model of alcohol-induced hepatic fibrosis. As expected, this signaling pathway was activated following the administration of alcohol, but was then inhibited following treatment with curcumin. Furthermore, TGF- $\beta / \mathrm{Smad}$ signaling seems indispensible in transforming HSCs from a quiescent state to an active one (40). TGF- $\beta$ induces the phosphorylatoin and nuclear translocation of Smad2 and Smad3 in HSCs (41). Additionally, it has been demonstrated that the activation of Smad3 is more important than that of Smad2 in the morphological and functional maturation of fibrogenic cells (39). It has been suggested that Smad7, another Smad protein, acts as an inhibitor of TGF- $\beta /$ Smad signaling by abrogating the effects of TGF- $\beta 1$ (42). In this study, the downregulation of Smad7 with the upregulation of TGF- $\beta 1$ and phosphorylated Smad3 was observed in the alcohol-stimulated HSCs, suggesting that the HSCs were activated by alcohol. By contrast, following the administration of curcumin, the TGF- $\beta /$ Smad signaling pathway was inhibited in the alcohol-stimulated HSCs, as characterized by the upregulation of Smad7 and the downregulation of TGF- $\beta 1$ and phosphorylated Smad3 expression, resulting in the impairment of collagen-I and fibronectin synthesis and secretion in the activated HSCs.

In conlcusion, the data from the present study indicate that curcumin exerts hepatoprotective effects against the development of alcoloho-induced hepatic fribosis by inhibiting the proliferation and promoting the apoptosis of HSCs. These effects are mediated through the activation of ER stress and the suppression of the TGF- $\beta /$ Smad signaling pathway.

\section{References}

1. Williams R: Global challenges in liver disease. Hepatology 44: 521-526, 2006.

2. Mandayam S, Jamal MM and Morgan TR: Epidemiology of alcoholic liver disease. Semin Liver Dis 24: 217-232, 2004.

3. Poli G: Pathogenesis of liver fibrosis: role of oxidative stress. Mol Aspects Med 21: 49-98, 2000.

4. Brenner DA: Molecular pathogenesis of liver fibrosis. Trans Am Clin Climatol Assoc 120: 361-368, 2009.

5. Senoo H: Structure and function of hepatic stellate cells. Med Electron Microsc 37: 3-15, 2004.

6. Gao B and Bataller R: Alcoholic liver disease: pathogenesis and new therapeutic targets. Gastroenterology 141: 1572-1585, 2011.

7. Siegmund SV, Dooley S and Brenner DA: Molecular mechanisms of alcohol-induced hepatic fibrosis. Dig Dis 23: 264-274, 2005.

8. Dooley S, Streckert M, Delvoux B and Gressner AM: Expression of Smads during in vitro transdifferentiation of hepatic stellate cells to myofibroblasts. Biochem Biophys Res Commun 283: 554-562, 2001

9. Derynck R and Zhang YE: Smad-dependent and Smadindependent pathways in TGF-beta family signalling. Nature 425: 577-584, 2003.

10. Shi Y and Massague J: Mechanisms of TGF-beta signaling from cell membrane to the nucleus. Cell 113: 685-700, 2003.

11. Kavsak P, Rasmussen RK, Causing CG, et al: Smad7 binds to Smurf2 to form an E3 ubiquitin ligase that targets the TGF beta receptor for degradation. Mol Cell 6: 1365-1375, 2000.

12. Chen K, Zhang S, Ji Y, et al: Baicalein inhibits the invasion and metastatic capabilities of hepatocellular carcinoma cells via down-regulation of the ERK pathway. PLoS One 8: e72927, 2013.

13. Wu L, Cai B, Zheng S, Liu X, Cai H and Li H: Effect of emodin on endoplasmic reticulum stress in rats with severe acute pancreatitis. Inflammation 36: 1020-1029, 2013. 
14. Geng J, Peng W, Huang Y, Fan H and Li S: Ginsenoside-Rg1 from Panax notoginseng prevents hepatic fibrosis induced by thioacetamide in rats. Eur J Pharmacol 634: 162-169, 2010.

15. O'Connell MA and Rushworth SA: Curcumin: potential for hepatic fibrosis therapy? Br J Pharmacol 153: 403-405, 2008.

16. Noorafshan A and Ashkani-Esfahani S: A review of therapeutic effects of curcumin. Curr Pharm Des 19: 2032-2046, 2013.

17. Tu CT, Yao QY, Xu BL, Wang JY, Zhou CH and Zhang SC: Protective effects of curcumin against hepatic fibrosis induced by carbon tetrachloride: modulation of high-mobility group box 1 , Toll-like receptor 4 and 2 expression. Food Chem Toxicol 50: 3343-3351, 2012

18. Yao QY, Xu BL, Wang JY, Liu HC, Zhang SC and Tu CT: Inhibition by curcumin of multiple sites of the transforming growth factor-beta1 signalling pathway ameliorates the progression of liver fibrosis induced by carbon tetrachloride in rats. BMC Complement Altern Med 12: 156, 2012.

19. Kim JM, Kim HG, Han JM, et al: The herbal formula CGX ameliorates the expression of vascular endothelial growth factor in alcoholic liver fibrosis. J Ethnopharmacol 150: 892-900, 2013

20. Bruck R, Ashkenazi M, Weiss S, et al: Prevention of liver cirrhosis in rats by curcumin. Liver Int 27: 373-383, 2007.

21. Wang Y, Cheng M, Zhang B, Nie F and Jiang H: Dietary supplementation of blueberry juice enhances hepatic expression of metallothionein and attenuates liver fibrosis in rats. PLoS One 8 e58659, 2013.

22. Traber PG, Chou H, Zomer E, et al: Regression of fibrosis and reversal of cirrhosis in rats by galectin inhibitors in thioacetamide-induced liver disease. PLoS One 8: e75361, 2013.

23. Su LJ, Chang CC, Yang CH, et al: Graptopetalum paraguayense ameliorates chemical-induced rat hepatic fibrosis in vivo and inactivates stellate cells and Kupfer cells in vitro. PLoS One 8: e53988, 2013.

24. Liu ZW, Zhu HT, Chen KL, et al: Protein kinase RNA-like endoplasmic reticulum kinase (PERK) signaling pathway plays a major role in reactive oxygen species (ROS)-mediated endoplasmic reticulum stress-induced apoptosis in diabetic cardiomyopathy. Cardiovasc Diabetol 12: 158, 2013.

25. Bataller R and Brenner DA: Liver fibrosis. J Clin Invest 115: 209-218, 2005

26. Lee UE and Friedman SL: Mechanisms of hepatic fibrogenesis. Best Pract Res Clin Gastroenterol 25: 195-206, 2011.

27. Padhye S, Chavan D, Pandey S, Deshpande J, Swamy KV and Sarkar FH: Perspectives on chemopreventive and therapeutic potential of curcumin analogs in medicinal chemistry. Mini Rev Med Chem 10: 372-387, 2010.
28. Sharma RA, Gescher AJ and Steward WP: Curcumin: the story so far. Eur J Cancer 41: 1955-1968, 2005.

29. Fu Y, Zheng S, Lin J, Ryerse J and Chen A: Curcumin protects the rat liver from $\mathrm{CCl} 4$-caused injury and fibrogenesis by attenuating oxidative stress and suppressing inflammation. Mol Pharmacol 73: 399-409, 2008.

30. Rivera-Espinoza Y and Muriel P: Pharmacological actions of curcumin in liver diseases or damage. Liver Int 29: 1457-1466, 2009.

31. Sun X, He Y, Ma TT, Huang C, Zhang L and Li J: Participation of miR-200a in TGF- 31 -mediated hepatic stellate cell activation. Mol Cell Biochem 388: 11-23, 2014.

32. Kodama T, Takehara T, Hikita H, et al: Increases in $\mathrm{p} 53$ expression induce CTGF synthesis by mouse and human hepatocytes and result in liver fibrosis in mice. J Clin Invest 121: 3343-3356, 2011.

33. Friedman SL: Evolving challenges in hepatic fibrosis. Nat Rev Gastroenterol Hepatol 7: 425-436, 2010.

34. Ron D and Walter P: Signal integration in the endoplasmic reticulum unfolded protein response. Nat Rev Mol Cell Biol 8: 519-529, 2007.

35. Rao RV, Ellerby HM and Bredesen DE: Coupling endoplasmic reticulum stress to the cell death program. Cell Death Differ 11: 372-380, 2004

36. Lee AS: The ER chaperone and signaling regulator GRP78/ $\mathrm{BiP}$ as a monitor of endoplasmic reticulum stress. Methods 35: 373-381, 2005.

37. Roberts AB, Tian F, Byfield SD, et al: Smad3 is key to TGF-betamediated epithelial-to-mesenchymal transition, fibrosis, tumor suppression and metastasis. Cytokine Growth Factor Rev 17: 19-27, 2006.

38. Leask A and Abraham DJ: TGF-beta signaling and the fibrotic response. FASEB J 18: 816-827, 2004.

39. Bissell DM, Roulot D and George J: Transforming growth factor beta and the liver. Hepatology 34: 859-867, 2001.

40. Schnabl B, Kweon YO, Frederick JP, Wang XF, Rippe RA and Brenner DA: The role of Smad3 in mediating mouse hepatic stellate cell activation. Hepatology 34: 89-100, 2001.

41. Liu C, Gaca MD, Swenson ES, Vellucci VF, Reiss M and Wells RG: Smads 2 and 3 are differentially activated by transforming growth factor-beta (TGF-beta) in quiescent and activated hepatic stellate cells. Constitutive nuclear localization of Smads in activated cells is TGF-beta-independent. J Biol Chem 278: 11721-11728, 2003.

42. Friedman SL:Mechanisms of hepatic fibrogenesis. Gastroenterology 134: 1655-1669, 2008. 